Arab Univ.

J. Agric. Sci., Ain Shams Univ., Cairo, 25(2), 355-365, 2017

\title{
ETIOLOGY OF FUNGI ASSOCIATED WITH GRAPEVINE DECLINE AND THEIR PATHOLOGICAL POTENTIAL
}

\author{
Hemida1, K.A.R.; E.H.E. Ziedan $^{1}$; M.G.M. El-Saman ${ }^{2}$; M.A. El-Naggar ${ }^{1}$ \\ and H.M. Mostafa ${ }^{2}$ \\ 1- Plant Pathology Dept., National Research Centre, Giza, Egypt \\ 2- Plant Pathology Dept., Faculty of Agriculture, Ain Shams University, Cairo, Egypt
}

Keywords: Grapevine, Root-rot, Etiology, pathological potential, Fusarium spp., Botryodiplodia theobromae, Rhizoctonia solani.

\section{ABSTRACT}

Decline of grapevine due to soil borne fungi was surveyed during 2013- 2015 summer growing seasons at El-Fayoum, El-Gharbeia and ElBeheira governorates, Egypt, Syndromes of declined grapevine plants included growth retardant of shoot system and root-rot as well as decrease of grapevine fruit yield quality. Isolation trails from root of declined trees of different grapevine cultivars i.e., superior, flame seedless, King robi and crimson was carried out. The most soil borne fungi associated with root-rotted grapevine were Fusarium oxysporum Schlechtend, Fusarium solani (Mart.) Sacc, Botryodiplodia theobromae, Rhizoctonia solani Kuhn and Macrophomina phaseolina(Tassi) Gold. Fusarium spp. are the main fungal associated with different infection types of declined root of grapevine either a singly or in combination with $B$. theobromae or $R$. solani as second infection type and third infection type with $B$. theobromae $+R$. solani. Under soil artificially infested with $5 \%(\mathrm{w} / \mathrm{w})$ of each fungal isolates inocula of fourteen isolates obtained were varied for causing wilt and root-rot symptoms of grapevine trees and reducing growth parameters plant height, root length, root size, fresh and dry weight of shoot and root than the control. Botrydiplodia theobromae isolate No. (7) was the most caused root-rot and disease severity of shoot and root of grapevine plant $(100 \%)$ followed by Fusarium avenacum caused $(87.5 \%)$ of root -rot and disease severity of shoot and root. Fouthermore, Fusarium avenacum isolate was the most fungal isolate in reducing growth parameters expect root length. This is first record that Fusarium avenacum as a causal organism causing root rot disease of grapevine in Egypt.

\section{INTRODUCTION}

Grapevine (Vitis vinifera L.) is one of the most widely distributed fruit crop in the world and the second fruit crop after citrus in Egypt. Total area cultivated of grapevine in Egypt are (192934) feddan yielded (1596169) ton of grapevine fruits. The largest cultivation areas at EL-Beheira followed by EL-Minia and EL- Gharbeia governorates respectively (Anonymous, 2015).

Decline of grapevine worldwide in the countries cultivation showing symptoms of decline and death of grapevine plants. The most common soil borne fungi causing decline of grapevine are Cylindrocarpon sp. (Grasso, 1985 and Gugino et al 2001), Fusarium oxysporum, Fusarium solani (Andrade et al 1995; Cruz et al 2014 and Gugino et al 2001), Rhizoctonia solani (Krol, 2006), Phytophthora sp., Macrophomina phaseolina (Van coller et al 2005), Botryospheria sp., Verticillium $\mathrm{sp}$. and Phacoacremonium sp. (Garrido et al 2004).

In Egypt grapevine is attacking by several soil borne pathogenic fungi causing wilt and root-rot diseases i.e. Fusarium oxysporum, Fusarium solani, Fusarium moniliforme, Rhizoctonia solani, Botryodiplodia theobromae and Macrophomina phaseolina (Badaway, 1973; Mourad, 1983; Mahrous, 1994; Ziedan, 2003; Ziedan et al 2005; Ziedan et al 2008; Ziedan et al 2011 and ElMorsi et al 2015).

The symptoms of wilt and root rot disease on grapevine began on bottom leaves chlorosis of lower and then these turned to necrotic spots and 
the leaves died and showing yellowing, wilting, stunting and necrotic lesions while discoloration was observed on root system, Wilt symptoms were spread to apical associated with vascular discoloration of roots and stem basal (Andrade et al 1995; Ziedan, 2003 and Ziedan et al 2011).

Little information of ecological relations between soil borne pathogenic fungi on grapevine. Meanwhile several were studied on some plants. On senna (Cassia acuitfolia Delile) synergistic effect between pathogenic isolates of $F$. oxysporum, $F$. solani and $R$. solani were recorded on pre and post emergency damping off than individual isolates (Zaher et al 1979). On sugar can F. moniliforme became more epidemic for induce stalk red rot than individual fungi in combination with Colletotrichum falcatum (Biswas and Samajpti, 1991). Mahrous (1994) reported that different combinations between fungi i.e. F. moniliformae, F. solani, $F$. tricincin, $F$. rosum, $R$. solani and $B$. theobromae causing root-rot of cutting grapevine were synergistic effect on disease incidence and other combinations were antagonistic than each one of fungi. Ziedan (2000) found that in the combination between F. oxysporum and Aspergillus niger significantly increased root-rot incidence of Peanut. Root-rot of japanase persimmon (Diospyros kaki L.) was increased in the combination between $M$. phaseolina $+F$. semitecum and $R$. solani $+F$. semitecum than each one individual (Ragab et al 1997).

Ziedan, (2003) found that on grapevine Fusarium spp., R. solani and M. phaseolina are causing root-rot of grapevine. Single infection model occur by Fusarium spp.or $R$. solani, M. phaseolina was not observed. Second infection model caused by Fusarium spp. $+R$. solani or Fusarium spp.+ $M$. phaseolina and three fungi was found in third model of infection. Third and second model more frequency then single model.

This study aimed to investigation of etiology and ecological relation between soil borne fungi causing decline of grapevine in Egypt.

\section{MATERIALS AND METHODS}

\section{Survey of fungal root diseases on grapevine}

In Egypt, during 2013-2015 summer seasons survey of decline grapevine orchards 7-10 years old was carried out at El-Fayoum, El-Behira and El- Gharbeia governorates. Survey was carried out on superior, flamseedless and crimson cultivars. Diseased grapevine trees exhibiting on shoot sys- tem yellowing, wilting, stunting, shortening and defoliation of twigs. Reduction of fruit yield components included number and weight of cluster. Syndromes i.e. necrotic lesions, maceration and discoloration of root system and stem base under soil ground. Percentages of diseased grapevine trees were recorded and disease severity of shoot and root were determined according to (Ziedan, 2003) as follows: $0=$ healthy, $1=$ yellowish $+1 / 3$ plant wilted, $2=2 / 3$ plant wilted, $3=$ whole plant wilted and $4=$ plants dead showed sever wilt.

\section{Isolation and identification of causal organisms}

Samples of diseased grapevine roots of crimson, flamseedless, superior and King robi cultivars were collected from El-Fayoum, El-Beheira and ElGharbeia governorates, Egypt. Root samples were thoroughly washed under running tap water then cut into small pieces $(1 \mathrm{~cm})$. These pieces were surface sterilized by dipping in $1 \%$ sodium hypochlorite solution for 2 min then washing several times by sterile distilled water. Pieces were dryed between two layers of sterilized filter paper then transferred individually to Petri dishes. Each plate contained $20 \mathrm{ml}$ potato dextrose agar (PDA) medium. Plates were incubated at $28^{\circ} \mathrm{C}$ for $5-7$ days. Developed fungal colonies were purified using hyphal tip or single spore techniques according to (Booth, 1971; Nelson et al 1983; Barnett and Hunter, 1986). Purified cultures of isolated fungi were identified according to cultural morphological and microscopically characteristics described by Nelson et al (1983) and Barnett and Hunter (1986). Pure culture isolates were maintained on PDA slants and kept in refrigerator at $5^{\circ} \mathrm{C}$ for further studies.

\section{Pathogenicity tests}

The pathogenic ability of fungal isolates were carried out under greenhouse conditions at Plant Pathology Department, National Research Centre, Egypt. Plastic pots $(25 \mathrm{~cm}$ - diameter) were sterilized by dipping in a $5 \%$ formalin solution for 15 min. Loam sandy soil was sterilized with formalin solution (5\%), and then covered with a polyethylene sheet for 7 days to retain the gas, and left to dry for 2 weeks until all traces of formaldehyde disappeared. Pots were filled with sterilized soil (3.5 $\mathrm{Kg} \mathrm{pot).} \mathrm{Each} \mathrm{isolate} \mathrm{of} \mathrm{tested} \mathrm{fungi} \mathrm{were}$ grown on sterilized corn meal and sand medium ( $75 \mathrm{~g}$ corn meal grain $+25 \mathrm{~g}$ clean pur sand $+100 \mathrm{ml}$ of water) of glass bottles. Each bottle was inocu- 
lated by disk ( $1 \mathrm{~cm}$ - diameter) taken from 7 daysold of mycelial cultures of each tested fungal isolates, then incubated at $27 \pm 2^{\circ} \mathrm{C}$ for 15 days. Pots were infested with each tested fungi by the rate $5 \%$ of soil weight $(\mathrm{w} / \mathrm{w})$. The pots were irrigated regularly three times a week before planting to ensure even distribution of the inoculated fungus in the soil. One grapevine transplant $\mathrm{Cv}$. crimson (one year old) was cultivated of each pot. Ten pots were used as replicates. Ten pots free fungi infestation were cultivated as a control. Percentage of root-rot disease incidence and disease severity were recorded 2.5 months after cultivation of grape plants. Disease severity was determined on shoot system of grape plant according to Ziedan, (2003) as follows: $0=$ healthy, $1=$ yellowish $+1 / 3$ plant wilted, $2=$ $2 / 3$ plant wilted, $3=$ whole plant wilted and $4=$ plants dead showed sever wilt. Also, root-rot disease severity of root was determined according to (Ziedan, 2003) on line scale from 0 to 3 as follows: $0=$ normal color (health), 1 = slight brown discoloration, 2 = moderate brown discoloration, 3 = dark brown discoloration.

\section{Estimation of grapevine plants morphological characters}

At the experimental end (2.5 months) after cultivation, morphological characters of grape plants in pots i.e. plant height, root length $(\mathrm{cm})$, fresh and dry weight $(\mathrm{g})$ of shoot and root and root size $\left(\mathrm{cm}^{3}\right)$ were determined according to (Ziedan, 1998).

\section{Statistical analysis}

Statistical analyses of all the previously designed experiments were carried out according to (ANOVA) procedures reported by Snedecor and Cochran (1982). Treatment means were compared by Duncan's multiple range test at $5 \%$ level of probability.

\section{RESULTS}

\section{Survey of decline on grapevine plants}

During summer seasons of 2013-2015 grapevine orchard 7-10 years old were inspected for decline syndromes. Growth retardant of shoot system, chlorosis, dieback, wilt and reduction of yield components i.e. number and weight of clusters and total yield of grapevine tree were determined. Root rot syndrome were observed included maceration and brown discoloration of feeder roots, secondary roots and stem base underground soil of grapevine of superior and flame seedless were observed at El-Fayoum and El-Behira governorates, Egypt. High percentage of diseased grapevine tree was found in El-Fayoum than in El-Beheira of grapevine (Cvs.) superior followed by flame seedless. The high number of clusters and total yield of grapevine tree during 2014 and 2015 were recorded of grapevine in El-Behira than El-Fayoum. Number of clusters of grapevine cultivars superior and flame seedless were from 2013 and fresh yield was recorded at each governorate.

Table 1. Survey of grapevine decline under field conditions

\begin{tabular}{|c|c|c|c|c|c|c|c|c|c|}
\hline \multirow{3}{*}{ Year } & \multirow{3}{*}{$\begin{array}{l}\text { Location } \\
\text { Cultivar }\end{array}$} & \multicolumn{4}{|c|}{ El- Fayoum } & \multicolumn{4}{|c|}{ El-Beheira } \\
\hline & & \multicolumn{2}{|c|}{ Root-rot disease } & \multicolumn{2}{|c|}{ Clusters } & \multicolumn{2}{|c|}{ Root-rot disease } & \multicolumn{2}{|c|}{ Clusters } \\
\hline & & infection\% & D.S & $\begin{array}{c}\text { number } \\
/ \text { tree }\end{array}$ & $\begin{array}{l}\mathrm{Kg} \mathrm{/} \\
\text { tree }\end{array}$ & infection\% & D.S & $\begin{array}{c}\text { number } \\
/ \text { tree }\end{array}$ & $\begin{array}{l}\mathrm{Kg} \mathrm{/} \\
\text { tree }\end{array}$ \\
\hline \multirow[t]{2}{*}{2013} & Superior & $48.0^{a}$ & $2.3^{\mathrm{ab}}$ & ND & ND & $40.0^{\mathrm{b}}$ & $2.0^{\mathrm{ab}}$ & ND & ND \\
\hline & $\begin{array}{l}\text { Flame } \\
\text { seedless }\end{array}$ & $40.0^{\mathrm{b}}$ & $2.2^{\mathrm{ab}}$ & ND & ND & $42.0^{b}$ & $2.4^{\mathrm{a}}$ & ND & ND \\
\hline \multirow[t]{2}{*}{2014} & Superior & $51.0^{\mathrm{a}}$ & $2.5^{\mathrm{a}}$ & $6.5^{\mathrm{ab}}$ & $4.2^{\mathrm{ab}}$ & $48.0^{\mathrm{ab}}$ & $2.0^{a b}$ & $7.0^{\mathrm{a}}$ & $5.0^{\mathrm{a}}$ \\
\hline & $\begin{array}{c}\text { Flame } \\
\text { seedless }\end{array}$ & $44.0^{\mathrm{ab}}$ & $2.4^{\mathrm{a}}$ & $7.0^{\mathrm{a}}$ & $4.3^{\mathrm{ab}}$ & $36.0^{\mathrm{b}}$ & $1.62^{b}$ & $7.0^{\mathrm{a}}$ & $5.2^{\mathrm{a}}$ \\
\hline \multirow[t]{2}{*}{2015} & Superior & $53.0^{a}$ & $2.8^{a}$ & $6.2^{\mathrm{a}}$ & $4.2^{\mathrm{ab}}$ & $48.0^{\mathrm{ab}}$ & $2.5^{\mathrm{a}}$ & $7.5^{\mathrm{a}}$ & $5.4^{\mathrm{a}}$ \\
\hline & $\begin{array}{c}\text { Flame } \\
\text { seedless }\end{array}$ & $50.0^{a}$ & $2.5^{\mathrm{a}}$ & 6. $0^{\mathrm{ab}}$ & $4.0^{\mathrm{ab}}$ & $42.0^{b}$ & $2.2^{\mathrm{ab}}$ & $6.8^{a}$ & $4.8^{\mathrm{a}}$ \\
\hline
\end{tabular}

$\mathrm{ND}=$ Not determined; $\mathrm{D} . \mathrm{S}=$ disease severity.

Mean values within columns followed by the same letter are not significantly different $(P \leq 0.05)$ according to Duncan's multiple range test. 


\section{Fungal infection of grapevine roots}

Percentage of grapevine root infected by different fungal genera was determined of superior and flame seedless cultivars at El-Fayoum governorate, Egypt at two side i.e. Tamia and Youssef El-Sedik distracts during 2014, Data presented in Table (2) indicated that routine isolation of diseased grapevine by root-rot of cultivars superior and flame seedless, yielded several fungal genera and species i.e. Aspergillus niger, Aspergillus flavus, Aspergillus terrus, Penicillum sp., Botrydiplodia theobromae, Fusarium spp., Rhizoctonia solan, Epicocum nigram, high percentage of root-rot infection was recorded by Fusarium spp. of all grapevine cultivars under this study in all location followed by Botryodiplodia theobromae .

In addition, data in Table (2) indicated that the high infection of grapevine root by saprophytic fungi i.e. Aspergillus spp., Penicillum sp. was recorded in Tamia, El-Fayoum. Meanwhile high percentage by pathogenic fungal isolates was by Fusarium spp., Botrydiplodia was observed in Youssef EL-Sedik, El-Fayoum of flamseedless followed by superior cultivars.

\section{Frequency of fungi associated of diseased grapevine roots}

Frequency of fungal genera of diseased grapevine root of superior and flame seedless cultivars was determined in two side at El-Fayoum governorate, Egypt during 2014. Data in Table (3) indicated that the high frequency of all fungal associated were found of superior cultivar in Tamia than Youssef El-Sedik distract. Fungal genera included Aspergillus niger with high frequency than other Aspergillus species i.e. A. flavus and $A$. terrus. The most high frequency of soil borne pathogenic fungi was observed Fusarium spp. followed by Botryodiplodia theobromae meanwhile the least frequency was found by Rhizoctonia solani.

\section{Root rot infection types of grapevine roots}

During 2014, routine isolation trails of diseased root rotten tissue of different grapevine cultivars i.e. superior, flame seedless, crimson and King robi were determined on El-Fayoum, El-Gharbeia and El-Behira governorates, Egypt .Four soil borne pathogenic fungi were found to associate with diseased roots i.e. as Fusarium spp., Botryodiplodia theobromae, Rhizoctonia solani and Macrophomina phaseolina. Data presented in Table (4) indicated that high percentage of single infection type was recorded by Fusarium spp. of all grapevine cultivars at all governorates followed by $B$. theobromae of superior at El-Fayoum governorate. On the other hand, no single infection type due to $R$. solani or M. phaseolina was observed.

Second combination of pathogenic fungi of grapevine by soil borne pathogenic fungi was Fusarium spp.+ Botryodiplodia theobromae on all grapevine cultivars at all governorates, followed by Fusarium spp. + $R$. solani which only one observed at El-Behira governorate on superior, flame seedless cultivars. The only one third combinations were recorded between Fusarium spp. + Botryodiplodia theobromae and $R$. solani on superior and flame seedless cultivars at El-Fayoum and ElBehira governorates. Meanwhile, no fourth infection types were recorded between four fungi on grapevine cultivars in this study.

\section{Pathogenicity test of fungal isolates of grapevine}

Fourteen isolates of fungi were isolated from different grapevine cultivars in El-Fayoum, ElGharbeia and El-Behira governorates, Egypt as shown in Table (5). All isolated fungi i.e. Fusarium spp., Botryodiplodia theobromae, Rhizoctonia solani and Macrophomina phaseolina were tested for their pathogenic potential on grapevine CV. crimson under potted infested soil with $5 \%$ of soil weight $(w / w)$ of each fungal isolates. Data presented in Table (5) and Fig. (1) indicated that root rot disease incidence was observed on grapevine plants meanwhile, vascular wilt symptoms was not observed on tested grapevine plants. Two patterns of root-rot symptoms were observed on shoot system of grapevine plants as shown in Fig. (2). Isolates of $F$. solani, B. theobromae, $R$. solani and $M$. phaseolina were induced chlorosis, yellowish on grapevine plant from bottom to top then plants wilted and died, meanwhile isolates of $F$. oxysporum and $F$. avenacum were induced mainly necrosis of leaf for along of shoot system. In addation all fungal isolates were reduced root system growth, secondary and feeder root was rotted as well as brown discoloration was observed Fig. (1) and Fig. (3). Root rot disease percentage and disease severity were recorded $30,60,75$ days after cultivation grapevine plants. 

pathological potential

Table 2. Percentage of repeating fungal colonization of grapevine diseased roots in El-Fayoum governorate during 2014

\begin{tabular}{|l|c|c|c|c|c|}
\hline \multirow{2}{*}{ Fungal name } & \multicolumn{4}{|c|}{ El- Fayoum } & \multirow{2}{*}{ Mean } \\
\cline { 2 - 5 } & \multicolumn{2}{|c|}{ Tamia } & Youssef El-Sedik & \\
\cline { 2 - 5 } & Superior & Flame seedless & Superior & Flame seedless & \\
\hline Aspergillus niger & 44.0 & 76.0 & 23.3 & 30.0 & 43.3 \\
Aspergillus flavus & 8.0 & 0.0 & 0.0 & 0.0 & 2.0 \\
Aspergillus terrus & 44.0 & 4.0 & 0.0 & 0.0 & 12.0 \\
Penicillum sp. & 12.0 & 0.0 & 0.0 & 0.0 & 3.0 \\
Botrydiplodia theobromae & 4.0 & 12.0 & 40.0 & 13.3 & 17.3 \\
Fusarium spp. & 60.0 & 32.0 & 76.7 & 88.3 & 64.2 \\
Rhizoctonia solani & 0.0 & 0.0 & 13.3 & 0.0 & 3.3 \\
Epicocum nigram & 0.0 & 0.0 & 0.0 & 10.0 & 2.0 \\
\hline
\end{tabular}

Table 3. Frequency of fungal associated with diseased grapevine roots in El-Fayoum governorate

\begin{tabular}{|c|c|c|c|c|c|}
\hline \multirow{4}{*}{ Fungal name } & \multicolumn{4}{|c|}{ Frequency of fungal colonies $\%$} & \multirow{4}{*}{ Mean } \\
\hline & \multicolumn{4}{|c|}{ El-Fayoum } & \\
\hline & \multicolumn{2}{|c|}{ Tamia } & \multicolumn{2}{|c|}{ Youssef El-Sedik } & \\
\hline & Superior & $\begin{array}{c}\text { Flame } \\
\text { seedless }\end{array}$ & Superior & $\begin{array}{c}\text { Flame } \\
\text { seedless }\end{array}$ & \\
\hline Aspergillus niger & 11.6 & 58.9 & 9.6 & 22.1 & 35.3 \\
\hline Aspergillus flavus & 6.8 & 0.0 & 0.0 & 4.8 & 2.9 \\
\hline Aspergillus terrus & 14.0 & 1.4 & 0.0 & 0.0 & 7.7 \\
\hline Penicillum sp. & 5.3 & 0.0 & 0.0 & 0.0 & 2.7 \\
\hline Botryodiplodia theobromae & 5.9 & 4.3 & 23.4 & 3.7 & 5.1 \\
\hline Fusarium spp. & 42.5 & 30.4 & 52.0 & 64.2 & 36.1 \\
\hline Rhizoctonia solani & 0.0 & 5.0 & 7.4 & 0.0 & 3.1 \\
\hline Epicocum nigram & 0.0 & 0.0 & 4.2 & 0.0 & 1.05 \\
\hline Mucor spp. & 13.9 & 0.0 & 13 & 5.2 & 7.78 \\
\hline
\end{tabular}

Table 4. Percentage of fungal infection types on different grapevine cultivars under natural field infestation

\begin{tabular}{|c|c|c|c|c|c|c|c|c|c|}
\hline \multirow{3}{*}{$\begin{array}{l}\text { Infection } \\
\text { type }\end{array}$} & \multirow{3}{*}{$\begin{array}{c}\text { Governorates } \\
\text { Fungi }\end{array}$} & \multicolumn{3}{|c|}{ El-Fayoum } & \multirow{2}{*}{\multicolumn{3}{|c|}{$\begin{array}{l}\text { El-Gharbia } \\
\text { EI-Mahalla }\end{array}$}} & \multirow{2}{*}{\multicolumn{2}{|c|}{$\begin{array}{l}\text { El-Behira } \\
\text { El-Nobaria }\end{array}$}} \\
\hline & & \multirow{2}{*}{$\begin{array}{c}\text { Tamia } \\
\text { Superior }\end{array}$} & \multicolumn{2}{|c|}{ Youssef El-Sedik } & & & & & \\
\hline & & & Superior & $\begin{array}{c}\text { flame } \\
\text { seedles }\end{array}$ & Crimson & $\begin{array}{l}\text { King } \\
\text { robi }\end{array}$ & $\begin{array}{c}\text { flame } \\
\text { Seedles }\end{array}$ & Superior & $\begin{array}{c}\text { Flam } \\
\text { seedless }\end{array}$ \\
\hline \multirow{4}{*}{ Single } & Fusarium spp. & 40 & 0.0 & 66.7 & 75 & 60 & 0.0 & 0.0 & 57.1 \\
\hline & B. theobromea & 20 & 0.0 & 0.0 & 0.0 & 0.0 & 0.0 & 0.0 & 0.0 \\
\hline & R. solani & 0.0 & 0.0 & 0.0 & 0.0 & 0.0 & 0.0 & 0.0 & 0.0 \\
\hline & M. phaseolina & 0.0 & 0.0 & 0.0 & 0.0 & 0.0 & 0.0 & 0.0 & 0.0 \\
\hline \multirow[t]{2}{*}{ Second } & $F+B$ & 40 & 66.7 & 33.2 & 25 & 40 & 100 & 0.0 & 14.2 \\
\hline & $F+R$ & 0.0 & 0.0 & 0.0 & 0.0 & 0.0 & 0.0 & 100 & 14.3 \\
\hline Third & $F+B+R$ & 0.0 & 33.3 & 0.0 & 0.0 & 0.0 & 0.0 & 0.0 & 14.3 \\
\hline
\end{tabular}

$\mathrm{F}=$ Fusarium spp., $\mathrm{B}=$ Botryodiplodia theobromae, $\mathrm{R}=$ Rhizoctonia solani, $\mathrm{M}=$ Macrophomina phaseolina 


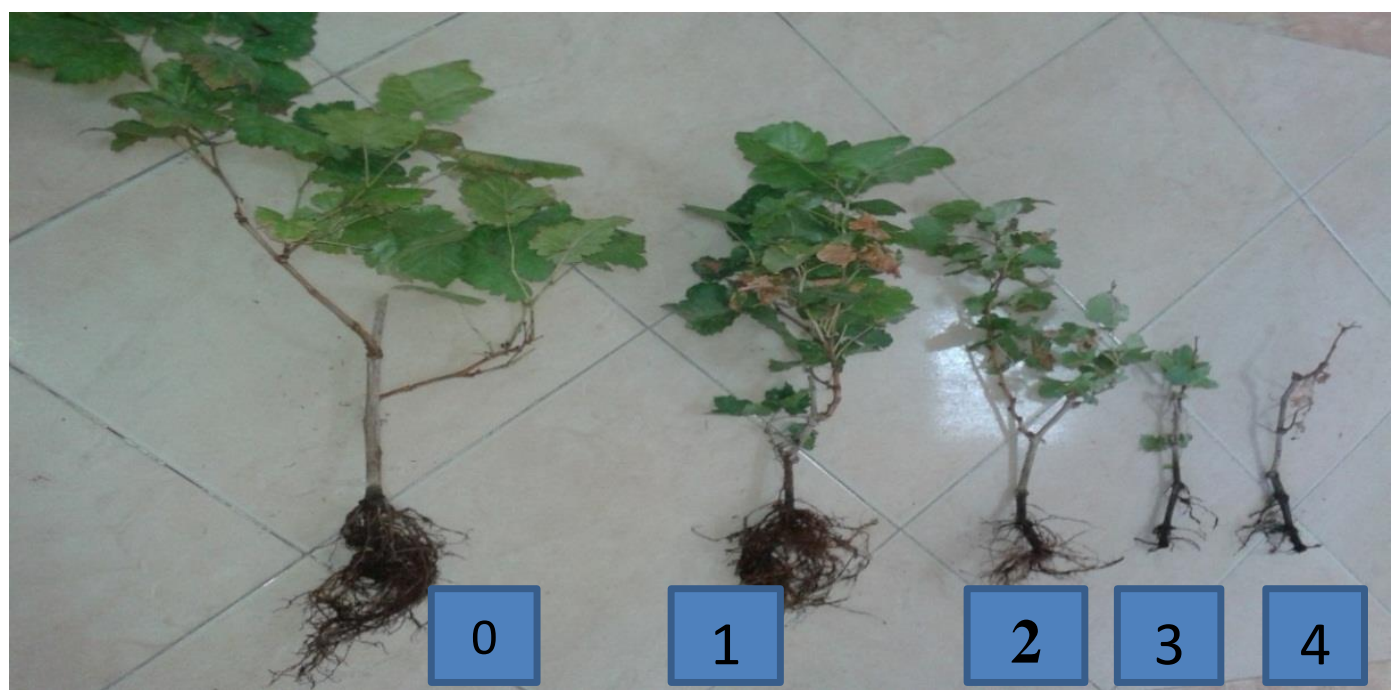

Fig. 1. Different root-rot disease severity of shoot and root of grapevine Cv. crimson, under artificial infested soil by soil borne fungi.

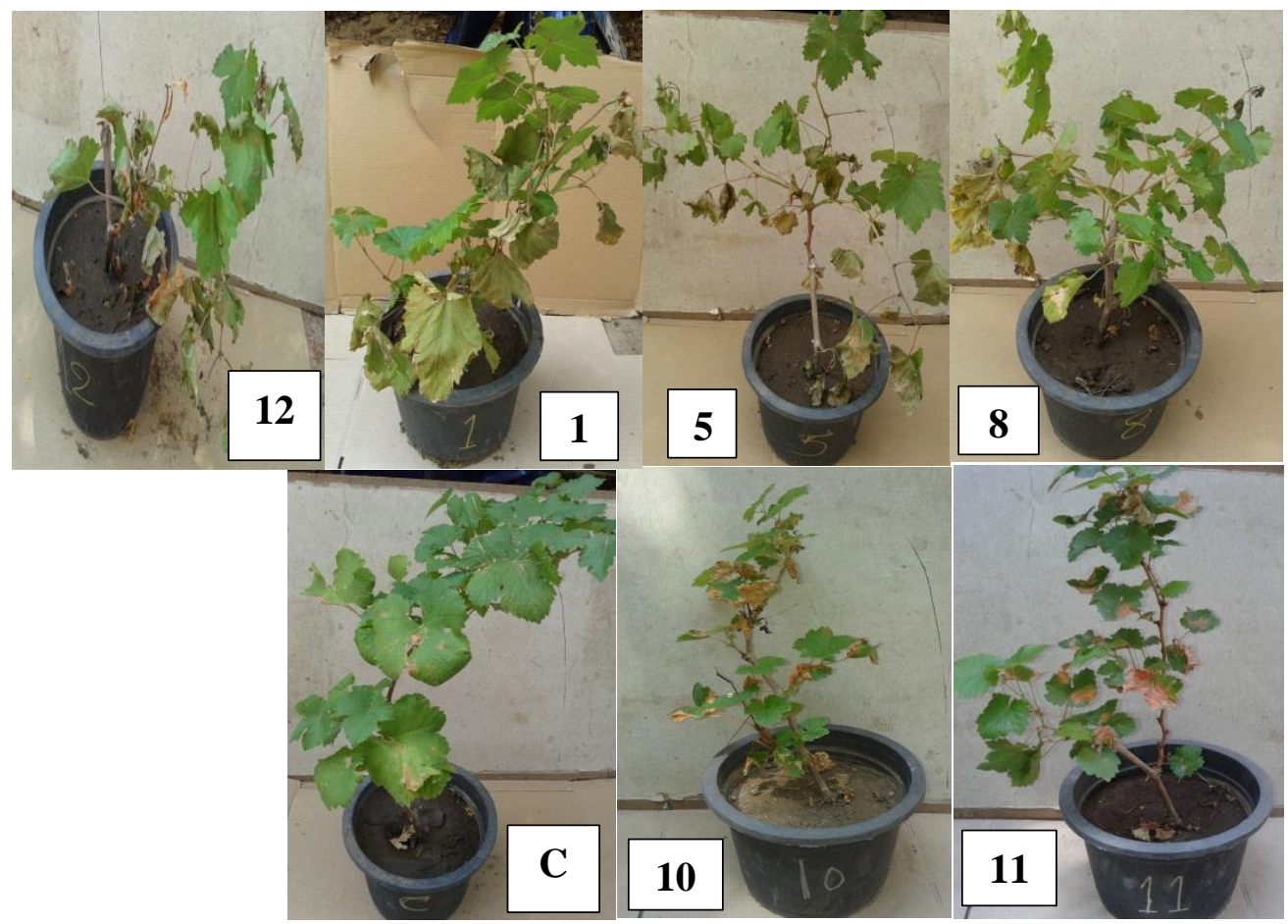

Fig. 2. Disease symptoms of grapevine on shoot system of Cv. crimson, under artificial infested soil showing chlorosis, yellowish, necrosis and wilt due to infection by isolates i.e. Botryodiplodia theobromae No.(1) Fusarium oxysporum No. (10), F. solani No. (12), R. solani No.(8) M. phaseolina No. (5) and Fusarium avenacum No. (11) compeer with control (c). 


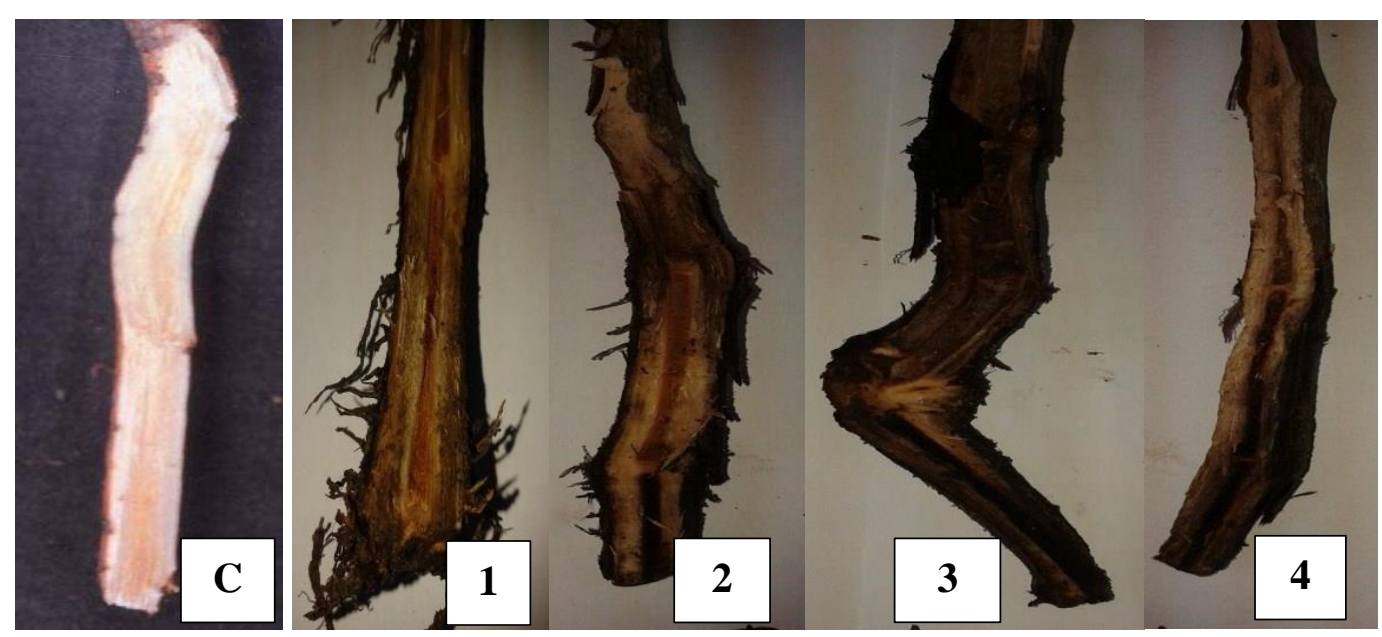

Fig. 3. Root-rot discoloration and maceration of grapevine root system of Cv. crimson, by Fusarium oxysporum (1), R. solani (2), Macrophomina phaseolina (3) and B. theobromae(4) compeer with control (c).

Table 5. Pathogenicity test of fungal isolates on grapevine plants cv. Crimson, sowin in artificily infested soil under greenhouse conditions

\begin{tabular}{|c|c|c|c|c|c|c|c|c|c|}
\hline \multirow{4}{*}{ 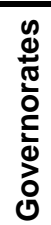 } & \multirow{2}{*}{\multicolumn{2}{|c|}{ Fungi }} & \multicolumn{7}{|c|}{ Root- rot incidence on grapevine } \\
\hline & & & \multicolumn{2}{|c|}{ After 30 days } & \multicolumn{2}{|c|}{ After 60 days } & \multicolumn{3}{|c|}{ After 75 days } \\
\hline & \multirow{2}{*}{ fungal name } & \multirow{2}{*}{ No. } & \multirow{2}{*}{$\begin{array}{c}\text { infection } \\
\%\end{array}$} & \multirow{2}{*}{$\begin{array}{l}\text { Disease } \\
\text { severity }\end{array}$} & \multirow{2}{*}{$\begin{array}{c}\text { infection } \\
\%\end{array}$} & \multirow{2}{*}{$\begin{array}{l}\text { Disease } \\
\text { severity }\end{array}$} & \multirow{2}{*}{$\begin{array}{c}\text { infection } \\
\%\end{array}$} & \multicolumn{2}{|c|}{$\begin{array}{l}\text { Disease } \\
\text { severity }\end{array}$} \\
\hline & & & & & & & & Shoot & root \\
\hline \multirow{5}{*}{ 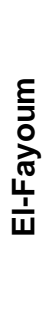 } & B.theobromae & 1 & $40 a b$ & $1.00 \mathrm{~b}$ & $60 \mathrm{bc}$ & $1.50 \mathrm{cde}$ & $75 c$ & $1.62 \mathrm{cde}$ & $1.75 c$ \\
\hline & R. solani & 2 & $40 a b$ & $1.25 a b$ & $60 \mathrm{bc}$ & $1.75 \mathrm{cde}$ & $75 c$ & $1.87 \mathrm{bcde}$ & $1.87 \mathrm{bc}$ \\
\hline & F. solani & 3 & $30 b$ & $1.25 \mathrm{ab}$ & $50 \mathrm{~cd}$ & $1.40 \mathrm{de}$ & $63 d$ & $1.40 \mathrm{e}$ & $1.50 \mathrm{c}$ \\
\hline & F.oxysporum & 4 & $30 b$ & $1.00 \mathrm{~b}$ & $40 d$ & $1.50 \mathrm{cde}$ & $50 e$ & $1.62 \mathrm{cde}$ & $1.75 c$ \\
\hline & M.phaseolina & 5 & $30 b$ & $1.00 \mathrm{~b}$ & $50 \mathrm{~cd}$ & $1.25 \mathrm{e}$ & $62 d$ & $1.25 e$ & $1.50 \mathrm{c}$ \\
\hline \multirow{5}{*}{ 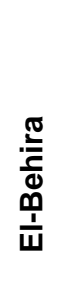 } & F. oxysporum & 6 & $30 b$ & $1.00 \mathrm{~b}$ & $60 \mathrm{bc}$ & 2.12abc & $87.5 b$ & $2.50 a b$ & $2.60 a b$ \\
\hline & B.theobromae & 7 & $50 a$ & $1.75 a$ & $80 a$ & $2.60 \mathrm{a}$ & $100 a$ & $2.87 a$ & $3.25 a$ \\
\hline & R. solani & 8 & $40 a b$ & $1.00 \mathrm{~b}$ & $60 \mathrm{bc}$ & $1.75 \mathrm{cde}$ & $87.5 \mathrm{~b}$ & $2.00 \mathrm{bcde}$ & 2.25abc \\
\hline & F. solani & 9 & $30 b$ & $1.40 \mathrm{ab}$ & $70 a b$ & 2.00abcd & $87.5 b$ & $2.50 \mathrm{ab}$ & 2.40abc \\
\hline & F. oxysporum & 10 & $40 a b$ & $1.25 a b$ & $70 a b$ & $1.75 \mathrm{cde}$ & $70 \mathrm{~cd}$ & $2.40 a b c$ & $2.50 \mathrm{abc}$ \\
\hline \multirow{4}{*}{$\begin{array}{l}\frac{\pi}{0} \\
\frac{0}{0} \\
\frac{\pi}{\pi} \\
\frac{1}{1} \\
\frac{1}{\omega}\end{array}$} & F. avenacum & 11 & $40 a b$ & $1.50 a b$ & $70 a b$ & $2.50 \mathrm{ab}$ & $87.5 b$ & $2.62 a b$ & $2.75 a b$ \\
\hline & F. solani & 12 & $40 a b$ & $1.50 a b$ & $70 a b$ & $2.12 \mathrm{abc}$ & $87.5 \mathrm{~b}$ & $2.50 a b$ & $2.62 \mathrm{ab}$ \\
\hline & B.theobromae & 13 & $30 b$ & $1.00 \mathrm{~b}$ & $60 \mathrm{bc}$ & 1.87bcde & $75 c$ & 2.12abcd & $2.12 b c$ \\
\hline & F. oxysporum & 14 & $40 a b$ & $1.60 \mathrm{a}$ & $60 \mathrm{bc}$ & $1.87 \mathrm{bcde}$ & $75 c$ & 2.00bcde & $2.12 b c$ \\
\hline \multicolumn{3}{|c|}{ Control } & $0.0 \mathrm{c}$ & $0.00 c$ & $0.0 \mathrm{e}$ & $0.00 f$ & $0.0 f$ & $0.00 f$ & $0.0 \mathrm{~d}$ \\
\hline
\end{tabular}

Means with the same letter are not significantly different $(P \leq 0.05)$ according to Duncan's multiple range test. 
Root rot incidence of grapevine was increased from 30, 60 and 75 days after cultivation. Fungal isolates from El-Behira recorded the highly root rot percentage and disease severity on grapevine plant followed by isolates of El-Gharbeia. Botryodiplodia theobromae No.(7) was the most fungal isolates recorded high and significant root rot percentage (100\%) and disease severity (2.9) and (3.3) on shoot and root respectively followed by Fusarium oxysporum isolate No.(6), then Fusarium avenacum No.(11) followed by $F$. solani No.(12) were recorded $(87.5 \%)$. F. oxysporum isolate No. (6) which isolated from (El- Nobaria) El- Behira governorate was the most Fusarium fungal isolates caused high root-rot percentage $(87.5 \%)$ and recorded high disease severity of shoot and root (2.5) and (2.6) respectively. On the other hand, isolates of El-Fayoum governorate, i.e. Fusarium oxysporum No. (4), F. solani No. (3) and $M$. phaseolina No. (5) were recorded the least percentage of root-rot and disease severity of grapevine plants.

\section{Effect of fungal isolates of grapevine plants growth parameters under greenhouse condi- tions}

Data in Table (6) indicated that, all isolates of fungal genera, Fusarium spp., $R$. solani, $M$. phaseolina and Botryodiplodia theobromae have significant effect on grapevine plant Cv. Crimson, 75 day after cultivation by each fungal in this study. All fungal isolates were reduced morphological characters i.e. length of shoot and root, fresh weight of shoot and root and root size of each treatment than the control.

F. oxysporum isolate No.(4), Botrydiplodia theobromae No.(7), Fusarium avenacum No.(11) from El-Behira governorate were the most fungal isolates reduced length of grapevine plants, Meanwhile two isolates of El-Gharbeia governorate i.e., Botryodiplodia theobromae No. (13) and F. oxysporum isolate No. (14) significantly reduced root length than other isolates. The high significant reduction of root size was recorded by $F$. oxysporum isolate No. (14) which isolated from El-Gharbeia governorate.

Table 6. Effect of fungal isolates on grapevine plants growth parameters sown in artificially infested soil under greenhouse conditions

\begin{tabular}{|c|c|c|c|c|c|c|c|c|c|}
\hline \multirow{3}{*}{ Governorates } & \multirow{2}{*}{\multicolumn{2}{|c|}{ Fungi }} & \multicolumn{7}{|c|}{ Growth parameters } \\
\hline & & & \multicolumn{2}{|c|}{$\begin{array}{l}\text { Length } \\
(\mathrm{cm})\end{array}$} & \multicolumn{2}{|c|}{$\begin{array}{l}\text { Fresh weight } \\
\text { (g) }\end{array}$} & \multicolumn{2}{|c|}{$\begin{array}{c}\text { Dry weight } \\
(\mathrm{g})\end{array}$} & \multirow{2}{*}{$\begin{array}{l}\text { Root } \\
\text { size } \\
\left(\mathrm{cm}^{3}\right)\end{array}$} \\
\hline & fungal name & No. & shoot & root & shoot & root & shoot & root & \\
\hline \multirow[t]{5}{*}{ El-Fayoum } & B.theobromae & 1 & $34.2 \mathrm{~b}$ & $20.7 a b$ & $25.5 a b$ & $17.6 a b$ & $9.9 b$ & 6.0abcde & $10.2 a b$ \\
\hline & R. solani & 2 & $41.0 a b$ & $19.5 b$ & $23.6 a b$ & $13.0 \mathrm{abc}$ & $7.9 b$ & $6.7 a b c d$ & $7.9 \mathrm{bcd}$ \\
\hline & F. solani & 3 & $40.0 a b$ & $19.2 b$ & 23.3ab & $13.5 \mathrm{abc}$ & $9.0 \mathrm{~b}$ & $5.2 \mathrm{bcde}$ & $9.6 a b c$ \\
\hline & F.oxysporum & 4 & $33.6 \mathrm{~b}$ & $15.0 \mathrm{~b}$ & $22.0 \mathrm{ab}$ & $11.0 \mathrm{bc}$ & $7.2 \mathrm{~b}$ & $4.5 \mathrm{bcde}$ & $7.2 \mathrm{bcd}$ \\
\hline & M.phaseolina & 5 & $42.5 a b$ & $16.0 \mathrm{~b}$ & $22.7 a b$ & $12.8 \mathrm{abc}$ & $7.87 b$ & $4.13 \mathrm{bcde}$ & $7.87 \mathrm{bcd}$ \\
\hline \multirow[t]{8}{*}{ El-Behira } & F. oxysporum & 6 & $36.0 \mathrm{~b}$ & $13.0 \mathrm{~b}$ & $15.8 a b$ & $10.25 b c$ & $6.4 b$ & 3.4de & $5.2 \mathrm{~cd}$ \\
\hline & B.theobromae & 7 & $31.0 \mathrm{~b}$ & $17.5 b$ & $22.3 a b$ & $12.87 a b c$ & $7.0 \mathrm{~b}$ & 6.4abcde & $7.75 \mathrm{bcd}$ \\
\hline & R. solani & 8 & $38.0 \mathrm{~b}$ & $13.0 \mathrm{~b}$ & $20.0 a b$ & $13.9 \mathrm{abc}$ & $8.4 b$ & 6.12abcde & $6.44 \mathrm{bcd}$ \\
\hline & F. solani & 9 & $39.4 b$ & $14.6 \mathrm{~b}$ & $18.9 a b$ & $13.0 \mathrm{abc}$ & $8.6 b$ & $6.94 a b c$ & $4.75 d$ \\
\hline & F. oxysporum & 10 & $40.0 a b$ & $15.2 b$ & $24.7 a b$ & $13.25 a b c$ & $8.65 b$ & 7.15ab & $4.95 d$ \\
\hline & F. avenacum & 11 & $31.0 \mathrm{~b}$ & $12.25 b$ & $13.0 \mathrm{~b}$ & $7.5 \mathrm{c}$ & $7.0 \mathrm{~b}$ & $2.8 \mathrm{e}$ & $3.75 d$ \\
\hline & F. solani & 12 & $34.0 \mathrm{~b}$ & $13.0 \mathrm{~b}$ & $18.2 \mathrm{ab}$ & $11.0 \mathrm{bc}$ & $8.2 b$ & $3.5 \mathrm{cde}$ & $4.5 d$ \\
\hline & B.theobromae & 13 & $38.75 b$ & $11.13 b$ & $24.4 a b$ & $13.0 \mathrm{abc}$ & $10.0 \mathrm{~b}$ & $4.6 \mathrm{bcde}$ & $5.0 \mathrm{~d}$ \\
\hline El-Gharbia & F. oxysporum & 14 & $37.7 \mathrm{~b}$ & $11.5 b$ & $20.5 a b$ & $10.4 b c$ & $9.2 b$ & $5.5 \mathrm{bcde}$ & $4.2 \mathrm{~d}$ \\
\hline \multicolumn{3}{|l|}{ Control } & $54.5 \mathrm{a}$ & $27.5 \mathrm{a}$ & $29.0 \mathrm{a}$ & $21.75 a$ & $14.5 \mathrm{a}$ & $10.7 \mathrm{a}$ & $13.8 \mathrm{a}$ \\
\hline
\end{tabular}

Means with the same letter are not significantly different $(P \leq 0.05)$ according to Duncan's multiple range test. 
In general, Fusarium avenacum No. (11) was the most and significantly fungal isolate recorded high reduction of most growth parameters i.e. shoot length, fresh and dry weight of shoot, root and root size.

\section{DISCUSSION}

Decline of grapevine orchard was increased by increasing age development due to dominant various soil borne pathogenic fungi Fusarium spp., Rhizoctonia solani, Macrophomina phaseolina and Botryodiplodia theobromae which cause wilt and root-rot disease, which significantly losses quality and quantity of fruit yield (Badaway, 1973; Mourad, 1983; Mahrous, 1994; Ziedan, 2003; Ziedan et al 2005; Ziedan and El-Mohamedy, 2008; Ziedan, et al 2011; Cruz et al 2014 and ElMorsi et al 2015).

In this study all isolated fungal isolates were caused root-rot of grapevine plants under artificial infested soil. Growth reduction of root system i.e. secondary and feeder roots was observed. Two patterns of root-rot symptoms were observed on shoot system. First pattern included chlorosis, yellowish and wilt from bottom to apical of grapevine shoot system caused by Fusarium spp., Rhizoctonia solani, Macrophomina phaseolina and Botryodiplodia theobromae. Second pattern included necrosis and wilt. This results are in agreement with (Mahrous, 1994 and Ziedan, 2003).

Little information about etiology and epidemiology of causal pathogen of grapevine due to latent infection of pathogenic fungi in apparently health plants. Latent infection still for a long time before developing disease symptoms. Hallen, et al (2003) In this respect recorded three model of infection types of root-rot causal organisms. First model by single genera caused mainly by Fusarium spp. of all grapevine cultivars at all various locations in this study followed by Botryodiplodia theobromae only on superior cultivar at El- Fayoum, governorate. No single infection type was recorded by either Rhizoctonia solani or Macrophomina phaseolina. Second model of infection types of grapevine root caused by Fusarium spp. + Botryodiplodia theobromae of all grapevine cultivars, at all locations with high frequency followed by Fusarium spp. + Rhizoctonia solani on superior and flamseedles at El-Beheira governorate. Third model of infection only caused by Fusarium spp. + Botryodiplodia theobromae + Rhizoctonia solani on superior at El-Fayoum and on flamseedles at El- Beheira gov- ernorates. This result are agreement with results obtained by Ziedan, (2003). In this study all fungal isolates associated with decline grapevine by rootrot and wilt symptoms are significantly causing root-rot and significantly reduced most morphological characters i.e. plant height, root length, fresh and dry weight of shoot and root as well as root size compare the control of grapevine plants Cv. Crimson i.e. Fusarium oxysporum, Fusarium avenacum, Fusarium solani, Botryodiplodia theobromae, Rhizoctonia solani and Macrophomina phaseolina. The highly root-rot incidence, disease severity and reduction of growth parameters of grapevine plants were obtained with isolates of Fusarium oxysporum, Fusarium avenacum and Botryodiplodia theobromae which isolated from ElBeheira governorate and Fusarium solani which isolated from El-Gharbeia governorate. These results are agreement with Badaway, (1973); Mourad, (1983); Mahrous, (1994) and El-Morsi et al 2015).

According to results obtained in this study rootrot of grapevine causing high losses in vineyard in nurseries, during growing and production, in the field, different fungal genera are causing decline of vineyards. With little information of ecological and pathological relation between causal organisms on grapevine. So development of different modern tools for knowledge and interpretations these relation will be increasing the efficiency of strategy programs of management wilt and root-rot diseases of grapevine.

\section{REFERENCES}

Andrade, E.R., Dal Bo, M.A., Schuck, E. and Gallotti, G.J.M. 1995. Evaluation of grapevine (Vitis spp.) resistance to Fusarium oxysporum f. sp. herbemontis in Rio depeixevalley, Santa Catarina State, Brazil. Acta Hortic Vitic Enol. 388, 65-69.

Anonymous 2015. Agricultural Statistical summer and Nil crops. 309 p.

Badawy, M.F. 1973. Studies on the decayed grape-cuttings in nursery in Egypt. M Sc. Fac. Agric., Al-Azhar Univ., Egypt. 76 p.

Barnett, H.L. and Hunter, B.B. 1986 Illustrated Genera of Imperfect Fungi. 4th ed., Macmillan Publishing Co., New York, USA, 218 p.

Biswas, A. and Samajpti, N. 1991. Study of ecological relationship of Colletotrichum falcatum with other fungi in sugar cane stalkes. Journal of Mycopathology Research, 29(1), 67-75. 
Booth, C. 1971. The genus Fusarium Common Wealth Mycological Institute, Kew. Surrey, England: $237 \mathrm{p}$.

Cruz, A.F., Pires, M.C., Soares, W.R.O., de Rezende, D.V. and Blum, L.E.B. 2014. Soilborne plant pathogens associated to decline of grapevine grown in greenhouse. J. Plant Physiology \& Pathology, 2(1), 1-6.

EL-Morsi, M.E.A., Abdel-Monaim, M.F. and Ahmad, E.F.S. 2015. Management of root rot and wilt diseases of date palm offshoots using certain biological control agents and its effect on growth parameters in the New Valley Governorate, Egypt, Journal of Phytopathology and Pest Management 2(1), 1-11.

Garrido, L. da R., Sonego, O.R. and Gomes, V. N. 2004. Fungi associated with grapevine showing decline and plant death in the state of Rio Grande do Sul, Southern Brazil, Fitopatologia Brasileira 29, 322-324.

Grasso, S. 1984. Infezioni di Fusarium oxysporum e di Cylindrocarpon destructans associate a una moria di giovani piante di vite in Sicilia. Inform Fitopatol 34, 59-63.

Gugino, B.K., Travis, J.W. and Stewart, E.L. 2001. Pathogenicity of Fusarium spp., Cylindrocarpon sp. and Diplodia sp. on grape roots. Phytopathology 91, Supplement: S34-39.

Halleen, F., Crous, P.W. and Petrini, O. 2003. Fungi associated with healthy grapevine cuttings in nurseries, with special reference to pathogens involved in the decline of young vines. Australas. Plant Pathol. 32, 47-52.

Krol, E. 2006. Fungi inhabiting decaying grapevine cuttings. J. of Plant Protection Research 46(4), 353-358.

Mahrous, H.A. 1994. Studies on root rot disease of grapevine. Ph.D. Thesis, Fac. Agric., Fayoum Univ., Egypt. 148 p.

Mourad, M.Y. 1983. Studies on root rot disease of grape in Egypt and its control. Ph.D. Thesis. Fac. Agric. Al-Azhar Univ., Egypt. 120 p.

Nelson, P.E., Toussoum, T.A. and Marasas, W.F.O. 1983. Fusarium spp. An Illustrated manual for identification. The Pennsylvania Univ. USA. 189 p.
Ragab, M.M., Soliman, N.E., Mourad, M.Y. and Abo Rehab, M.E. 1997. Root-rot of Japanese persimmon in Egypt. Egyptian Journal Phytopathology, 25(1), 37-43.

Snedecor, G.W. and Cochran, G.W. 1982. Statistical Methods, lowa State University Press, Ames, lowa, USA, $7^{\text {th }}$ ed. 125 p.

Van coller, G.J., Denman, S., Lamprecht, S.C. and Crous, P.W. 2005. New perspective on soil borne diseases of grapevines in nurseries. (internet cited 2011 Apr. 6) A. www.wynboer.co.za /recent articles.

Zaher, E.A., Abdelal, H.R., Ibrahim, A.N. and Ez El-Din, A.Y. 1979. Studies on root-rot disease of Senna (Cassia acutifolia) Egyptian Journal Phytopathology, 11(1-2), 1-11.

Ziedan, E.H. 1998. Integrated control of wilt and root-rot diseases of sesame in A. R. E., Ph.D. Thesis, Fac. Agric., Ain Shams Univ. 165 p.

Ziedan, E.H. 2000. Soil treatment with biofertilizers for controlling Peanut root and pod rot diseases in Nobaria province. Egyptian Journal Phytopathology, 28 (1-2), 17-26.

Ziedan, E.H. 2003. Root-rot diseases of grapevine in Egypt. J. Agric. Sci. Mansoura Univ., 28(2), 147-1481.

Ziedan, E.H.E. and El-Mohamedy R.S.R. 2008. Application of Pseudomonas fluorescens for controlling root-rot disease of grapevine. Research Journal of Agriculture and Biological Sciences, 4, 346-353.

Ziedan, E.H.E., Farrag, (Eman) S., ElMohamedy, R.S. and AbdAlla, M.A. 2010. Streptomyces alnias a biocontrol agent to rootrot of grapevine and increasing their efficiency by biofertilisers inocula. Archives Phytopathology Plant. Prot., 43, 634-646.

Ziedan, E.H.E., Embaby E.S.M. and Farrag, (Eman) S. 2011. First record of Fusarium vascular wilt on grapevine in Egypt. Archives of Phytopathology and Plant Protection, 44, 1719-1727.

Ziedan, E.H., Saad, (Moataza) M. and Farrag, (Eman) S. 2005. Biological control of grapevine root rot with antagonistic microorganisms. African Mycology and Biotechnology, 13(3),1936. 